\title{
Reduction of Implant RF Heating Through Modification of Transmit Coil Electric Field
}

\author{
Yigitcan Eryaman, Burak Akin, and Ergin Atalar*
}

In this work, we demonstrate the possibility to modify the electric-field distribution of a radio frequency (RF) coil to generate electric field-free zones in the body without significantly altering the transmit sensitivity. Because implant heating is directly related to the electric-field distribution, implantfriendly RF transmit coils can be obtained by this approach. We propose a linear birdcage transmit coil with a zero electric-field plane as an example of such implant-friendly coils. When the zero electric-field plane coincides with the implant position, implant heating is reduced, as we demonstrated by the phantom experiments. By feeding RF pulses with identical phases and shapes but different amplitudes to the two orthogonal ports of the coil, the position of the zero electricfield plane can also be adjusted. Although implant heating is reduced with this method, a linear birdcage coil results in a whole-volume average specific absorption rate that is twice that of a quadrature birdcage coil. To solve this issue, we propose alternative methods to design implant-friendly RF coils with optimized electromagnetic fields and reduced whole-volume average specific absorption rate. With these methods, the transmit field was modified to reduce RF heating of implants and obtain uniform transmit sensitivity. Magn Reson Med 65:1305-1313, 2011. (C) 2010 Wiley-Liss, Inc.

Key words: safety of MRI; transmit RF coils; RF heating; implant heating

Magnetic resonance imaging (MRI) is known as a very safe imaging technology. However, because of the possibility of inducing excessive currents on the metallic wires, MRI scanning is generally not performed on people with metallic implants such as pacemakers. A radio frequency (RF) electric field, although undesirable, is often generated in the body during the excitation of spins with RF magnetic-field pulses. Power absorbed by the body under this electric field is determined by the specific absorption rate (SAR) and needs to be kept at a level that is safe to the patients. If a patient with a metallic implant is examined using MRI, a very significant SAR amplification may occur around the implant, which may cause excessive body heating and burns. Because of this well-known problem, patients with metallic implants are currently not allowed inside the MRI scanners.

Department of Electrical and Electronics Engineering, Bilkent University, Ankara, Turkey.

Grant sponsor: TUBITAK; Grant number: 107E108.

${ }^{*}$ Correspondence to: Ergin Atalar, National Magnetic Resonance Research Center (UMRAM) and Department of Electrical and Electronics Engineering, Bilkent University, Ankara 06800, Turkey.

E-mail: ergin@ee.bilkent.edu.tr

Received 10 June 2010; revised 12 October 2010; accepted 17 October 2010.

DOI 10.1002/mrm.22724

Published online 8 December 2010 in Wiley Online Library (wileyonlinelibrary.com).

(C) 2010 Wiley-Liss, Inc.
Previous studies $(1,2)$ have assessed the implant heating problem via both in vitro and in vivo approaches. Mathematical models have also been presented $(3,4)$, and the validity of these models has been further verified by comparison with the experimental data. A detailed analysis of the problem was conducted in (3) by solving the bioheat equation with Green's function and the linear system theory. The maximum steady-state temperature increase in the tissue near a transmitter catheter antenna was calculated. In (4), a parameter called the "safety index" was introduced, which combines the effect of the SAR gain of the implant lead and the bioheat transfer process to measure the in vivo temperature changes. Variations of the safety index with respect to the length and radius of the implant lead, the thickness of the insulation, and tissue conductivity and permittivity were also investigated. These studies presented a good model of tissue heating caused by metallic wires in RF fields. In another study, experimental methods were developed to measure and monitor the RF-induced currents inside implants (5).

Modifications of the implant leads and wires for reducing the RF-induced heating were investigated in other studies. In two of these studies, a series of chokes was added to the coaxial cables $(6,7)$ to reduce the currents generated on the cable shield. In another study (8), the effects of coiled wires on induced heating were investigated. By introducing air gaps and lowering the parasitic capacitance, the self-resonance frequency of a coiled wire was shifted to the operating frequency. This change increased the impedance of the wire and, thus, reduced the RF heating. However, all of these designs are based on modifying the lead wires or cables, which makes it difficult to produce mechanically robust leads. In addition, for patients who already have pacemakers, replacing the original leads with these modified safer leads may not always be feasible. For these reasons, modifications of the implant lead designs or the catheters may not always be the most appropriate solution for the MRI-induced RF heating of metallic wires.

The relationship between the electric-field distribution and the temperature increase of the implant leads was recently investigated (9). It was found that tissue heating depends on the orientation of the lead with respect to the direction of the electric field. To identify the worstcase scenario, an optimization-based approach was used in (10) to calculate the EM field that could produce the maximum heating at the wire tip. However, studies to optimize the EM transmitter field to minimize the implant heating were not carried out.

In this study, we showed that the transmitter coil field used in MRI could be optimized to steer the electric field 
away from the implant lead and thus prevent heating. As demonstrated experimentally, a linearly polarized birdcage coil could be used for this purpose. Although this approach preserved the homogeneous transmit sensitivity characteristics of the coil, it caused a doubling of the whole-body SAR. To alleviate this problem and achieve uniform sensitivity, we further modified the field of the transmitter coil to minimize both whole-body SAR and the implant heating. Details of this approach are described in the following sections.

\section{THEORY}

Implant-friendly RF Coil

In the standard quadrature birdcage coils, the electric field is uniform in the angular direction but varies roughly linearly in the radial direction (11). Therefore, an implant lead placed at the edge of the body experiences a high-electric field, which induces currents both on the lead and in the body and eventually causes local SAR amplification. The electric-field distribution of a standard forward-polarized quadrature birdcage coil in an infinitely long homogeneous model can be approximated by ignoring the end-ring currents as follows (see the Appendix for a detailed derivation):

$$
E_{z}=\frac{-H_{\mathrm{f}} \omega \mu_{0} \rho}{2} e^{j \phi}, \quad E_{\phi}=0, \quad E_{\rho}=0
$$

where $E_{z}, E_{\phi}$, and $E_{\rho}$ are the longitudinal, angular, and radial components of the electric field, respectively; $H_{\mathrm{f}}$ is the transmit sensitivity of the coil; $\omega$ is the Larmor frequency; $\mu_{0}$ and $\varepsilon$ are the permeability and permittivity, respectively; $\sigma$ is the conductivity of the homogeneous model; $\rho$ and $\phi$ are the radial and angular coordinates in the cylindrical coordinate system, respectively; and $j$ is the imaginary number defined by $\sqrt{-1}$. Similarly, the electrical field of a linearly polarized coil can be expressed as follows:

$$
E_{z}=-H_{\mathrm{f}} j \omega \mu_{0} \rho \sin \phi, \quad E_{\phi}=0, \quad E_{\rho}=0
$$

Note that $j$ indicates a $90^{\circ}$ phase shift with respect to a real field expression. As can be seen from the above equations, linear and quadrature birdcage coils have similar transmit sensitivity yet different electric-field distributions. The transmit sensitivities of each coil are approximately uniform in the transverse plane. (Please see the Appendix for the detailed derivation.) It can be noted from the above equation that the electric field is zero over the entire $\phi=0$ plane. This plane can be steered into any angular direction by either changing the feeding location or simply rotating the linear birdcage coil. The same task can also be performed by controlling the amplitudes of the currents fed into the two ports of a quadrature birdcage coil. For example, if port-1 and port2 are set in such a way as to make the corresponding electric fields zero at the $\phi=0$ and $\phi=\pi / 2$ planes, respectively, then the excitation currents with relative amplitudes of $\cos \phi_{0}$ and $\sin \phi_{0}$ at ports 1 and 2 would generate a zero electric-field plane at $\phi=\phi_{0}$. Note that $\phi$ $=\phi_{0}$ plane covers both $\phi=\phi_{0}$ and $\phi=\phi_{0}+\pi$ half planes. If an implant lead lies on the zero electric-field plane, there will be no induced currents on the lead. Because setting the electric field to zero makes the perpendicular component of the magnetic field vanish at the same plane, this method also intrinsically prevents the H-field coupling.

Using a linear birdcage coil solves the heating problem for an arbitrarily shaped implant lead when the lead is located in the zero electric-field plane. Despite this modification of the electric-field distribution, the transmit sensitivity is not significantly disturbed. However, as previously shown (12), linear birdcage coils are not efficient for RF transmission when the volume average SAR is considered. For linear excitation, a reverse polarizedfield component coexists with the forward polarized component, and the whole-volume average SAR per unit flip angle is doubled.

\section{Transmit Field Optimization}

To alleviate the doubling problem of the whole-volume average SAR, a general formulation was developed. Because transmit sensitivity is determined by the forward polarized component of the magnetic field and SAR is determined by the electric-field distribution, the above-mentioned problem can be solved by optimizing the electromagnetic (EM) field of the coil (13). In this study, we successfully demonstrated the feasibility of such a strategy by obtaining the desired EM-field distribution in the body. Although the work is not trivial, once an optimum EM field is identified, it is possible to design a coil that produces the desired field. The design of such an optimum implant-friendly coil is left for a future study.

First, we assumed that the optimization would be conducted in a uniform cylindrical object. This assumption simplified the formulation but could also be used with the other geometries. Because a cylindrical object is assumed, the cylindrical basis functions were used to expand the optimum field solution that minimized the whole volume average SAR $(14,15)$ :

$$
\overrightarrow{\boldsymbol{E}}=\sum_{m=-\infty}^{\infty} \sum_{n=-\infty}^{\infty} \boldsymbol{E}_{m n} \cdot \overrightarrow{\boldsymbol{\alpha}}_{m n} \cdot e^{j m \phi} e^{-j \beta_{z} z}
$$

where $\phi$ and $z$ are the angular and $z$ coordinates in the cylindrical coordinate system, respectively, and $m$ and $n$, respectively, denote the index of the circumferential and longitudinal modes used in the basis expansion. $\boldsymbol{E}_{m n}$ is a $3 \times 2$ matrix that contains the electric-field basis functions for the $\rho, \phi$, and $z$ components as shown below:

$$
\boldsymbol{E}_{m n}=\left[\begin{array}{cc}
\boldsymbol{J}_{m}\left(\boldsymbol{\beta}_{\rho n} \boldsymbol{\rho}\right) & 0 \\
\frac{1}{\boldsymbol{\rho} \boldsymbol{\beta}} \boldsymbol{J}_{m}\left(\boldsymbol{\beta}_{\rho n} \boldsymbol{\rho}\right) & \frac{\boldsymbol{\omega} \boldsymbol{\mu}}{\boldsymbol{\beta}} \boldsymbol{J}_{m}^{\prime}\left(\boldsymbol{\beta}_{\rho n} \boldsymbol{\rho}\right) \\
-\boldsymbol{j} \boldsymbol{J}_{m}^{\prime}\left(\boldsymbol{\beta}_{\rho n} \boldsymbol{\rho}\right) & \frac{1}{\boldsymbol{\sigma}^{\prime} \boldsymbol{\rho}} \boldsymbol{J}_{m}\left(\boldsymbol{\beta}_{\rho n} \boldsymbol{\rho}\right)
\end{array}\right]
$$

$\boldsymbol{E}_{m n}$ is a function of $\rho$, the radial coordinate, but not of $\phi$ or $z \cdot \overrightarrow{\boldsymbol{\alpha}}_{m n}$ is a $2 \times 1$ vector whose elements are the constants that multiply the basis functions and $\overrightarrow{\boldsymbol{\alpha}}_{m n}=\left[\boldsymbol{A}_{m n} \boldsymbol{B}_{m n}\right]^{T}$. 
The transmit coil sensitivity can be expressed by evaluating the forward polarized field, which can be written in the summation form as

$$
H_{f}(\overrightarrow{\boldsymbol{r}})=\sum_{m n} \overrightarrow{\boldsymbol{H}}_{f m n}(\overrightarrow{\boldsymbol{r}}) \cdot \overrightarrow{\boldsymbol{\alpha}}_{m n} \cdot e^{j(m+1) \phi} e^{-j \beta_{z n} z}
$$

Each separate mode for $H_{\mathrm{f}}$ can be expressed as follows:

$$
\overrightarrow{\boldsymbol{H}}_{f m n}(\overrightarrow{\boldsymbol{r}})=\frac{1}{\sqrt{2}}\left[\frac{\boldsymbol{j} \boldsymbol{\sigma}^{\prime}}{\boldsymbol{\beta}_{\boldsymbol{\rho}}}, \frac{\boldsymbol{\beta}_{z n}}{\boldsymbol{\beta}_{\rho n}}\right] J_{m+1}(\beta \rho)
$$

where $\beta_{z n}=\frac{2 \pi}{L} n, \beta_{\rho n}^{2}=\beta^{2}-\beta_{z n}^{2}$, and $\sigma^{\prime}=\sigma+j \omega \varepsilon$ (15). Here, $\sigma^{\prime}$ denotes the complex conductivity of the medium, and $\beta_{z n}$ and $\beta_{\rho n}$ are the wave numbers along the radial and longitudinal directions, respectively, which can be calculated as $\beta^{2}=-j \omega \mu_{0}\left[\sigma+j \omega_{0} \varepsilon\right]$. $L$ is the length of the cylinder.

For $k$ points of interest, the whole summation in the $H_{f}$ expression can be written in the following matrix form:

$$
\boldsymbol{H} \boldsymbol{\alpha}=c
$$

where $\boldsymbol{c}$, the desired transmit sensitivity profile, is represented by a $k \times 1$ vector whose elements are equal to the desired $H_{f}$ values at each point of interest. $\alpha$ is a column vector that contains the weighting coefficients $\left(A_{m n} B_{m n}\right)$ for each separate mode. $\boldsymbol{H}$ is the transmit sensitivity matrix whose elements are equal to the basis functions of $H_{f}$ evaluated at the desired points of interest. $\boldsymbol{H}$ is a $k \times$ $(2 \times M \times N)$ matrix, where $M$ and $N$ denote the total number of circumferential $(m)$ and longitudinal $(n)$ modes that are used in the basis expansion. Implementing different field variations would require using different combinations of the cylindrical modes. To express a field exhibiting a rapid spatial change in the circumferential or the longitudinal direction, one must use higherorder modes in that particular direction. Therefore, to characterize an arbitrary EM field with this expansion, an infinite number of modes is required. For practical purposes, the number of modes is truncated in our study.

The desired target transmit sensitivity is one of the linear constraints for minimizing the average SAR. A separate constraint also exists on the electric field to reduce the implant heating.

To achieve the zero-implant-heating condition, the components of the electric field that are parallel to the lead should be set to zero. Therefore, the induced current on the lead wire will be zero. This condition can also be expressed as a linear constraint, similar to $H_{\mathrm{f}}$, as shown below:

$$
E \boldsymbol{\alpha}=\overrightarrow{\boldsymbol{O}}
$$

where $\overrightarrow{\boldsymbol{O}}$ is a $p \times 1$ vector with all of its elements equal to zero, and $p$ denotes the number of sample points where the electric field is set to zero. $\boldsymbol{E}$ is a $k \times(2 \times M$ $\times N$ ) matrix, where $M$ and $N$ denote the total number of circumferential $(m)$ and longitudinal $(n)$ modes that are used in the basis expansion. $\boldsymbol{E}$ matrix contains the basis functions for $E_{z}, E_{\phi}$, and $E_{\rho}$ evaluated at the desired zero electric-field locations.

The constraints on $H_{\mathrm{f}}$ and the components of the electric field can be combined into a single matrix equation: $\boldsymbol{F} \boldsymbol{\alpha}=\boldsymbol{e}$, where $\boldsymbol{F}$ and $\boldsymbol{e}$ are formed by concatenating the matrices $\boldsymbol{B}, \boldsymbol{E}$ and the vectors $\boldsymbol{c}$ and $\overrightarrow{\boldsymbol{0}}$, respectively. While it is desirable to set the magnetic and electric field to certain values at points of interest, the SAR needs to be under control. The expression for the average SAR is:

$$
\mathrm{SAR}=\sigma / M \int_{\text {body }}|E|^{2} d v
$$

where $\sigma$ the conductivity, $M$ is the total body mass and $\mathrm{d} v$ is the differential volume element. With the cylindrical mode expansion for a homogeneous body model, the resulting relation can be written as:

$$
\mathrm{SAR}=(\sigma / M) \times \sum_{m n} \overrightarrow{\boldsymbol{\alpha}}_{m n}^{H} \cdot \boldsymbol{R}_{m n} \cdot \overrightarrow{\boldsymbol{\alpha}}_{m n}
$$

where $\boldsymbol{R}_{m n}$ is a Hermitian matrix and can be computed using the following expression (15):

$$
\boldsymbol{R}_{m n}=\left[2 \pi \boldsymbol{L} \boldsymbol{\sigma} \int_{\boldsymbol{0}}^{\boldsymbol{r}} \boldsymbol{E}_{m n}^{H} \cdot \boldsymbol{E}_{m n} \rho d \rho\right]
$$

where $r$ denotes the radius of the homogeneous model. The average SAR can be expressed in a more compact form as $\boldsymbol{\alpha}^{\star} \boldsymbol{R} \boldsymbol{\alpha}$, where $\boldsymbol{R}$ is the electric-field cross-correlation matrix whose block diagonals are equal to $\boldsymbol{R}_{m n}$.

Among the infinite number of solutions satisfying $\boldsymbol{F} \boldsymbol{\alpha}$ $=\boldsymbol{e}$, the solution with the minimum volume average SAR can be found by minimizing $\boldsymbol{\alpha}^{\star} \boldsymbol{R} \boldsymbol{\alpha}$. The solution for $\alpha$ can then be defined as:

$$
\boldsymbol{\alpha}_{\mathrm{opt}}=\boldsymbol{R}^{-1} \boldsymbol{F}^{*}\left(\boldsymbol{F} \boldsymbol{R}^{-1} \boldsymbol{F}^{*}\right)^{-1} \boldsymbol{e}
$$

The minimum whole body SAR value can be computed as:

$$
\mathrm{SAR}_{\min }=\boldsymbol{e}^{*}\left(\boldsymbol{F} \boldsymbol{R}^{-1} \boldsymbol{F}^{*}\right)^{-1} \boldsymbol{e}
$$

These equations give the minimum possible SAR under the conditions of the desired transmit sensitivity and zero electric field near the implant. They also give the corresponding weights for the cylindrical expansion modes. Although this solution does not directly specify the type of coil to be used, $\boldsymbol{\alpha}_{\text {opt }}$ uniquely determines the EM field of the optimum coil. The significance of this result can be appreciated by experiments and simulations as explained in the next section.

\section{EXPERIMENTS AND SIMULATIONS}

Implant-friendly RF Coil

To demonstrate the proposed theory, heating of metallic wires with both linear and quadrature excitation was tested. A phantom head model of $16 \mathrm{~cm}$ in diameter and $25 \mathrm{~cm}$ in length was prepared with commercially available gel (Dr Oetker Jello, Izmir, Turkey). To measure conductivity and relative permittivity, a cylindrical 

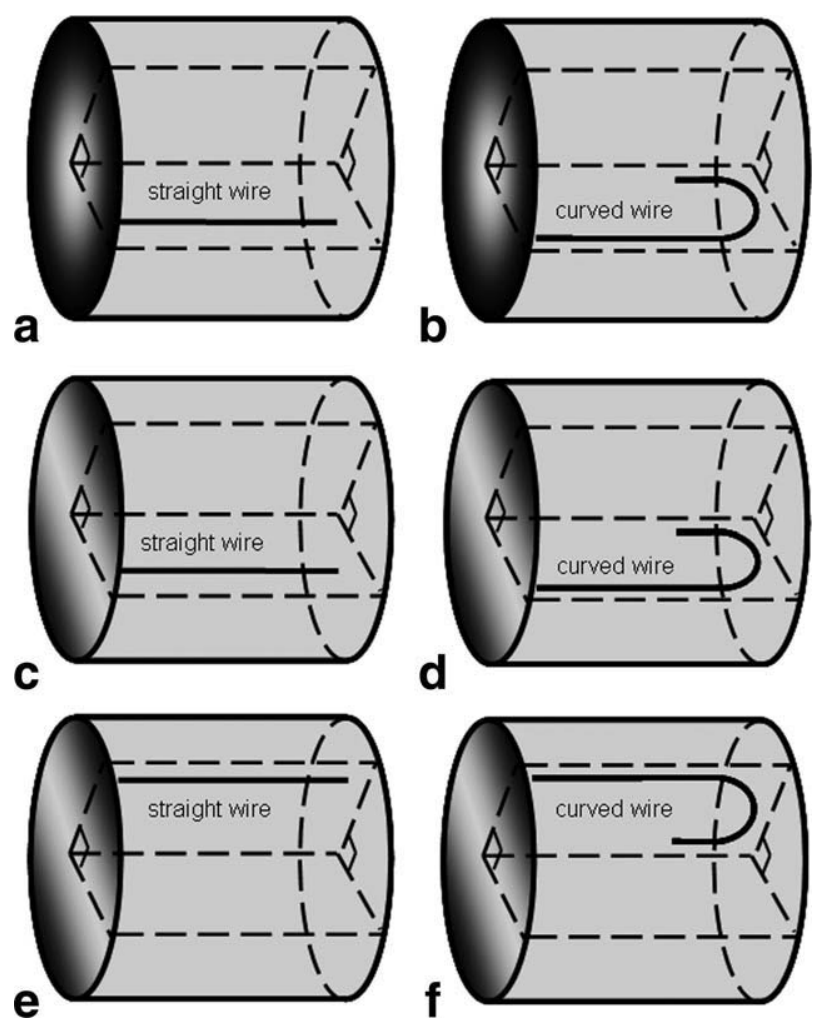

FIG. 1. Gel phantoms with straight and curved wires. Fiber-optic temperature measurements were performed near the tips of the lead wires. a and b: quadrature excitation; (c) and (d): linear excitation under the minimum heating condition; and (e) and (f): linear excitation under the maximum heating condition.

transmission line setup was used. By measuring the impedance at the end of the line and using the lossy transmission line impedance equations, the conductivity and relative permittivity were calculated (16). A conductivity of $0.51 \mathrm{~S} / \mathrm{m}$ and relative permittivity of 70 were obtained with $2.4 \mathrm{~g} / \mathrm{L}$ of salt in the gel solution. Heating experiments were performed with a straight wire and a curved wire, as shown in Fig. 1. Both of the wires were tested with quadrature and linear excitation.

The body coil of the Siemens 3.0 T Trio system was used in all experiments. A gradient echo sequence with a 4-msec TR and a $45^{\circ}$ flip angle was used to scan the phantoms. A peak SAR value of $4.4 \mathrm{~W} / \mathrm{kg}$ was obtained by finding the initial slope of the temperature rise and then multiplying it by the specific heat capacity of the gel, which was measured as $4100 \mathrm{~J} / \mathrm{kg} / \mathrm{deg}$ by using the KD2 Pro Thermal Properties Analyzer (Decagon Devices Inc., WA). The temperature measurement was conducted at a depth of $1 \mathrm{~cm}$ from the phantom surface.

When the phantom was scanned with the quadrature excitation, the temperature variations near the wire tips were recorded using a Neoptix ReFlex signal conditioner equipped with T1 fiber optic temperature sensors (Neoptix Inc., Quebec, Canada). The fiber optic probes were placed in a specific way so as to ensure contact with the wire tips. The temperature data for each lead were obtained from different scans. To ensure a fair compari- son, the gel phantom was kept in the refrigerator and allowed to reach the same initial temperature $\left(5.5^{\circ} \mathrm{C}\right)$. This low-initial temperature, rather than the room temperature of $19^{\circ} \mathrm{C}$, was chosen to prevent the gel from melting because it would be exposed to high heat during the experiment. The rate of temperature increase caused by heat conduction from the surface was approximately $2^{\circ} \mathrm{C} / \mathrm{h}$, which was significantly lower than that caused by the applied electric field.To obtain a linearly polarized excitation, one of the ports was disconnected. The orientation of the phantom was adjusted to make the location of the lead coincide with the zero electric-field plane. Once the temperature data under this condition (minimum heating condition) was collected, the phantom was rotated $90^{\circ}$ to position the lead in the maximum electricfield plane. Similar steps were taken for the measurement of curved wires. In all of the experiments, a single temperature probe was used to eliminate probe calibration errors and measurement errors caused by improper probe placement.

\section{Transmit Field Optimization}

The linearly polarized birdcage coils may solve the RF heating problem of the implant leads. As previously mentioned, a linear birdcage coil can generate a wholevolume averaged SAR that is twice that generated by a quadrature birdcage coil, which may be unacceptable for certain applications. Therefore, alternative implantfriendly strategies that can guarantee similar or better MR image homogeneity need to be identified.

As previously mentioned, instead of designing novel coils, we tried to optimize the electric-field distributions of currently available coils via simulation.

The optimization was conducted on a cylindrical head model with a conductivity of $0.5 \mathrm{~S} / \mathrm{m}$, relative permittivity of 70, diameter of $16 \mathrm{~cm}$, and length of $25 \mathrm{~cm}$. Four separate optimum field solutions were computed under four different sets of conditions, as given below.

\section{Quadrature Birdcage Coil}

The field distribution of an ideal quadrature coil was obtained using the above-mentioned optimization algorithm but with no constraint on the electric field. In this calculation, only a single point at the center of the object was chosen as the point of interest. Because of angular symmetry, the solution contained a single circumferential mode that corresponded to the field of a forward polarized birdcage coil. The whole-head averaged SAR calculated using this method can be considered as the minimum SAR one can obtain with a birdcage coil.

\section{Linear Birdcage Coil}

The field of the linearly polarized birdcage coil was directly constructed from the previous solution by introducing a reverse circular polarization mode. The conjugates of the field expansion coefficients calculated for the quadrature coil were used for the reverse polarized mode. According to our theory, this solution should contain a zero electric-field plane. If this field coincides with the plane of the implant lead, no implant heating 


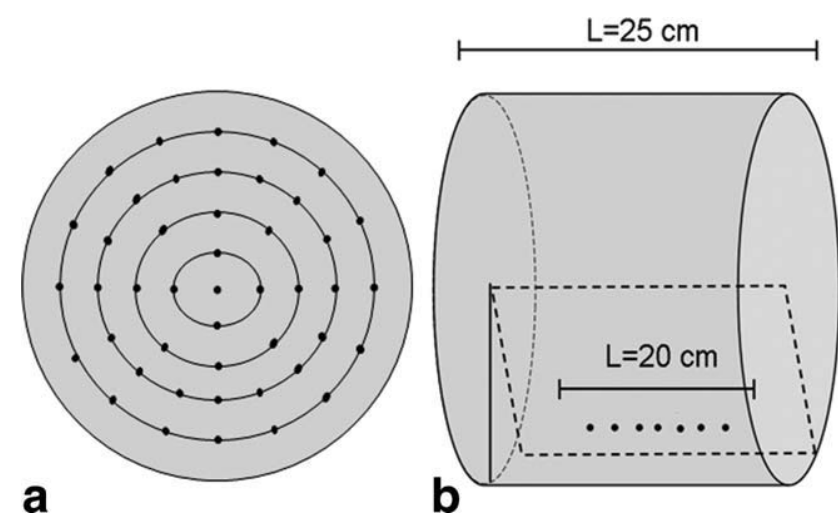

FIG. 2. To ensure homogeneous excitation, the coil transmit sensitivity was constrained to unity at 45 sample points, forming a circular region with a diameter of $15 \mathrm{~cm}$ on the transverse plane (a). The electric field was constrained to zero at seven sample points on a straight line whose distances to the phantom surface were 1 cm (b).

will be observed. Although this linearly polarized coil may be regarded as an implant-friendly coil, the wholehead average SAR obtained using this solution is twice as large as that of the quadrate birdcage coil. Therefore, a better solution is needed.

\section{Implant-friendly Coil}

To minimize the electric field around the implant, the exact location of the implant lead needs to be known. For demonstration purposes, a 20-cm straight implant lead is assumed to be placed $1 \mathrm{~cm}$ away from the surface in the longitudinal direction (Fig. 2).

Coil transmit sensitivities were chosen to be exactly the same as those for the linear birdcage coil. The optimization problem is solved by applying these transmit sensitivity values at sample points shown in Fig. 2. The tangential component of the electric field was set to zero at sample points on a lead, as shown in Fig. 2. This strategy guaranteed that the whole-head average SAR would be either less than or at most equal to the SAR of a linear birdcage coil because the transmit sensitivity was preserved, but the electric field constraints were relaxed.

\section{Implant-friendly Homogeneous Coil}

In an MRI scan, it is usually desirable to obtain a homogeneous transmit sensitivity in the region of interest. To achieve this goal, the transmit coil sensitivity is constrained to unity at points in the transverse plane (Fig. 2). The tangential component of the electric field was set to zero at sample points on a lead (Fig. 2). Results obtained from the above-mentioned experiments and simulations are given in the next section.

\section{RESULTS}

\section{Implant-friendly RF Coil}

Figure 3 shows the time course of the temperature increase near the tip of a straight wire in three different modes: the quadrature mode, the linear mode (maximum heating case), and the linear mode (minimum heating

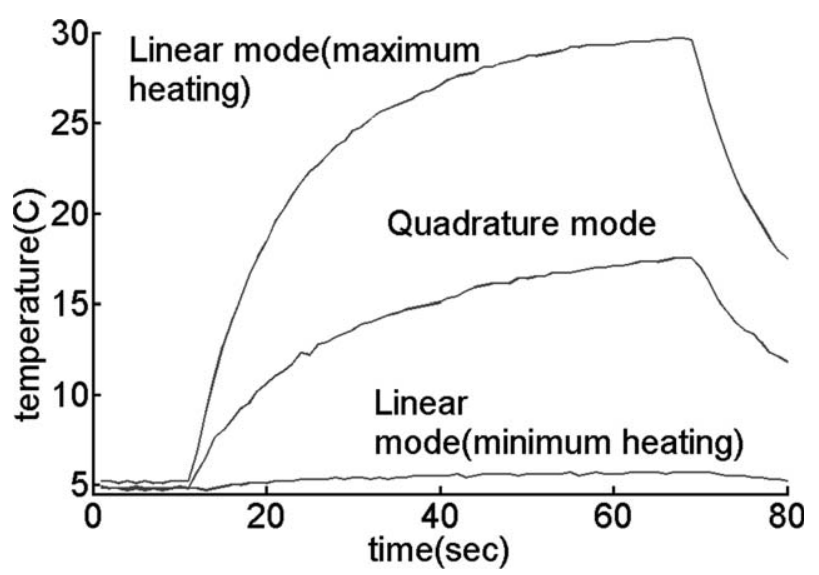

FIG. 3. Temperature rise as a function of time measured for a straight wire with three modes: the minimum heating linear mode, the maximum heating linear mode, and the quadrature mode. Final temperature increases of $0.8^{\circ} \mathrm{C}, 24.7^{\circ} \mathrm{C}$, and $12.1^{\circ} \mathrm{C}$ were observed with the minimum heating linear mode, the maximum heating linear mode, and the quadrature mode, respectively.

case). A temperature increase of $12.1^{\circ} \mathrm{C}$ was observed at the tip of the wire for the quadrature case. For the maximum heating case of the linear mode, an increase of $24.7^{\circ} \mathrm{C}$ was observed, For the minimum heating case of the linear mode, only a $0.8^{\circ} \mathrm{C}$ difference was observed, which was significantly smaller than the difference observed for the quadrature case. For the curved wires, similar trends were observed, with temperature increases of $9.2,19.1$, and $0.3^{\circ} \mathrm{C}$ observed for the three cases (Fig. 4). Again, the temperature change for the minimum heating case was significantly smaller than for the other two cases.

\section{Transmit Field Optimization}

To alleviate the whole body SAR problem due to a linearly polarized birdcage coil, the problem can be solved in its most general form by minimizing the whole body

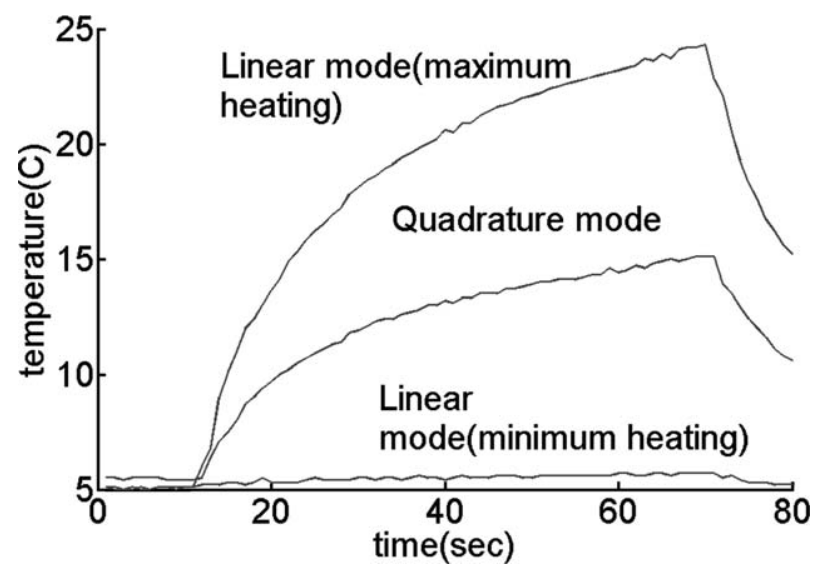

FIG. 4. Temperature rise as a function of time measured for a curved wire with three modes: the minimum heating linear mode, the maximum heating linear mode, and the quadrature mode. Final temperature increases of $0.3^{\circ} \mathrm{C}, 19.1^{\circ} \mathrm{C}$, and $9.2^{\circ} \mathrm{C}$ were observed with the minimum heating mode, the maximum heating linear mode, and the quadrature mode, respectively. 


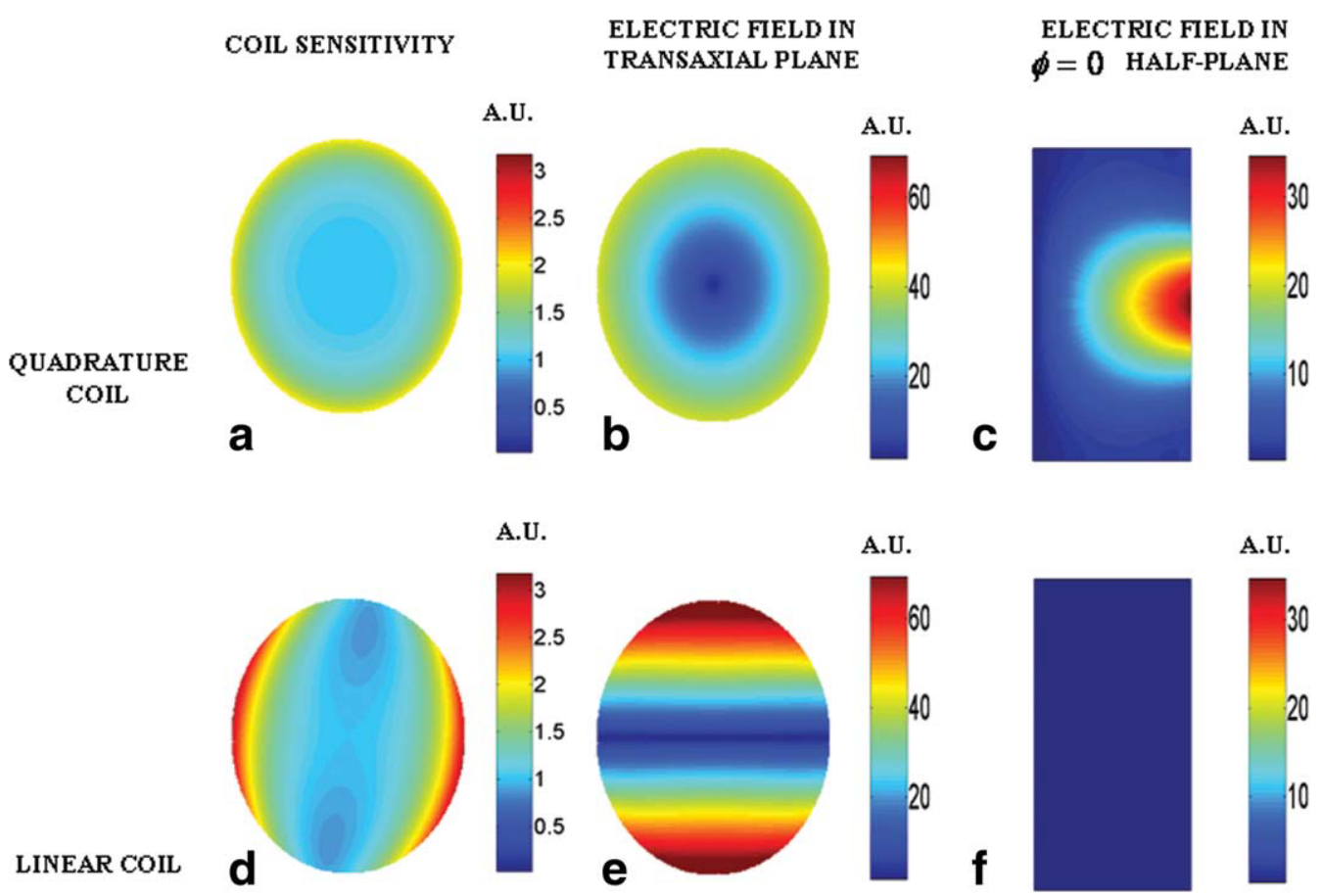

FIG. 5. Transmit sensitivity (a and $\mathbf{d}$ ), electric field in the trans-axial plane (b and $\mathbf{e}$ ) and electric field in the $\phi=0$ half-plane (c and $\mathbf{f}$ ) generated by quadrature and linear coils. Note that all field solutions are in arbitrary units.

SAR. The ranges of cylindrical mode index integers, $m$ and $n$, were chosen as $[-8,8]$ and $[10,10]$, respectively. These ranges were determined by running a convergence analysis on the average SAR value. It was numerically found that increasing the range of $m$ and $n$ further, does not change the solution more than $5 \%$. For transmit-field optimization, four different optimum field solutions were simulated under four different conditions as
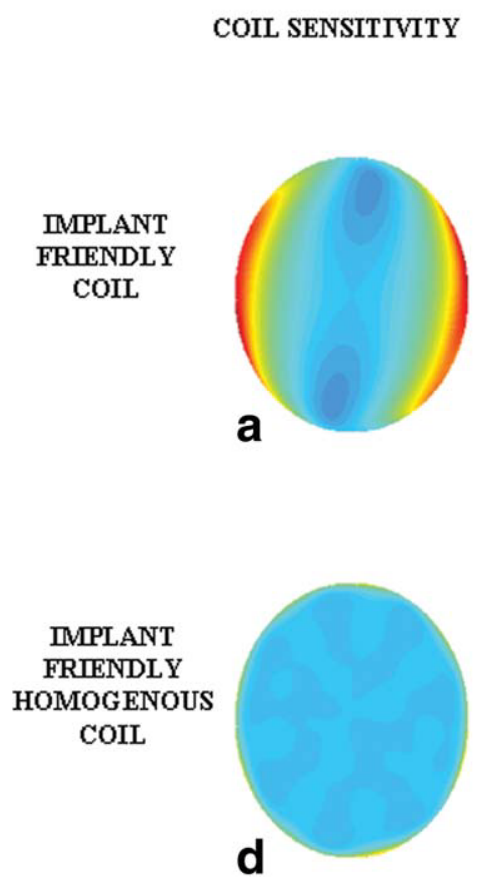

A.U.

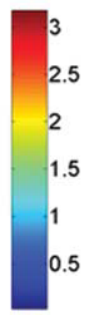

A.U.

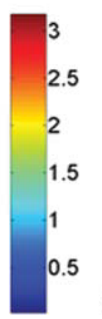

ELECTRIC FIELD IN TRANSAXIAL PLANE

A.U.

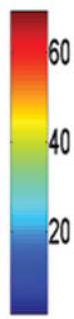

A.U.

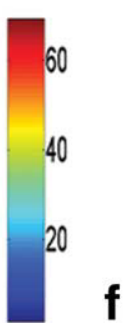

ELECTRIC FIELD IN

$\phi=0$ HALF-PLANE

FIG. 6. Transmit sensitivity ( $\mathbf{a}$ and $\mathbf{d}$ ), electric field in the trans-axial plane (b and $\mathbf{e}$ ) and electric field in the $\phi=0$ half-plane (c and $\mathbf{f}$ ) generated by implant-friendly coils and implant-friendly homogeneous coils. Locations of the implant lead are denoted by arrows in the figures. Note that all field solutions are in arbitrary units. 
described earlier. The transmit sensitivity of the midpoint is set to unity for all simulations. A homogeneity coefficient was calculated for each field solution by averaging the difference between the sensitivity of each pixel and the midpoint in the transverse plane. Note that a zero homogeneity coefficient represented a perfectly homogeneous transmit sensitivity profile.

\section{Quadrature Birdcage Coil}

Figure 5a shows the transmit sensitivity of the birdcage coil. The longitudinal components of the electric field on the trans-axial plane and on the " $\phi=0$ " half-plane are shown in Fig. 5b and c. The resulting minimum whole-head average SAR was also calculated. Because of angular symmetry, this solution imposed a single circumferential mode that corresponded to a perfectly forward circular-polarized field distribution. The wholehead average SAR value was defined as 1 au (arbitrary unit) in this case, which actually set the lower bound for the whole-head average SAR because $H_{\mathrm{f}}$ is constrained only to the origin. SAR values of the rest of the coils were defined accordingly. The homogeneity coefficient of this solution was found to be 0.41 . As can be seen from Fig. 5a, there is an approximately twofold difference between the sensitivity at the center and at the periphery. This variation is due to the conductive losses in the sample and to the wavelength effects.

\section{Linear Birdcage Coil}

A linearly polarized field was obtained from the previous solution as explained earlier. Figure $5 \mathrm{~d}$ shows the transmit sensitivity of the linear birdcage coil. The longitudinal components of the electric field on the transaxial plane and on the " $\phi=0$ " half-plane are shown in Fig. 5e and f. This field solution featured a zero electricfield plane, which made it a safer choice in terms of RF implant heating for implants in this plane. The sensitivity was equal to 1 at the origin. The SAR value obtained by this field configuration was twice as much as that of the quadrature birdcage coil. The linear coil did not have the same transmit sensitivity as the quadrature coil, but its homogeneity coefficient, calculated as 0.46 , was similar to that of the quadrature coil.

\section{Implant-friendly Coil}

Figure 6a shows the sensitivity of the implant-friendly coil. The longitudinal components of the electric field on the trans-axial plane and on the " $\phi=0$ " half-plane are shown in Fig. 6b and c. The longitudinal component of the electric field was forced to be equal to zero only around the implant lead. However, as can be seen from the figures, the electric field was zero on the lead and close to zero at the rest of the $\phi=0$ plane. The relaxed constraints on the electric field caused a decrease in the whole-head average SAR down to 1.49 au. The implantfriendly coil had a field solution that was very similar to the linear birdcage coil. The homogeneity coefficient of this solution, calculated to be 0.46 , equaled that of the linear coil.

\section{Implant-friendly Homogeneous Coil}

Figure 6d shows the sensitivity of the implant-friendly homogeneous coil. The longitudinal components of the electric field on the trans-axial plane and on the " $\phi=0$ " half-plane are shown in Fig. 6e and f. The whole-head SAR value obtained for this case was 2.96 au, which was significantly larger than those obtained from previous cases. On the other hand, the homogeneity coefficient of this solution was 0.0233 , which implied the possibility of building an implant-friendly coil with close to perfect homogeneity. However, under this circumstance, an increase in the average SAR became unavoidable.

\section{DISCUSSION}

It was shown in this study that a linear birdcage coil can be used as a transmitter coil in MRI and enable safe scanning of patients with implants. A linear birdcage coil has a transmit sensitivity similar to that of a quadrature birdcage coil. Moreover, the electric-field distribution of the linear coil is zero at an angular plane, and any metallic implants placed at this plane will experience zero heating. The angle of this plane can be adjusted to any angle $\phi=\phi_{0}$ by supplying appropriate excitation currents weighted as $\cos \phi_{0}$ and $\sin \phi_{0}$ in two ports of the linear birdcage coil. This task can be performed with a two-channel transmit array system, which is commercially available for some MRI scanners.

In all the experiments and simulations, homogeneous cylindrical head models were used. For real-life situations, variations in tissue conductivity may introduce some errors into the field calculations. To prevent implant RF heating, regions free of electric fields need to be created around the implant. In this study, we successfully generated such regions using special implantfriendly coils such as a linear birdcage coil; however, the performance of these designs needs to be further tested in patients.

In this work, it was theoretically shown that it is possible to find a field distribution that minimizes RF heating of implant leads. This was experimentally shown for two specific lead geometries that was confined in a cylindrical plane. For arbitrary lead geometries, the applicability of the method should also be investigated experimentally.

In a previous study (11), the electric-field distribution of a linearly polarized coil was calculated for a head model, and the existence of the zero electric-field region was demonstrated for field strengths up to $7.0 \mathrm{~T}$. It was also shown that, in the head model, the linear excitation had a homogeneous transmit sensitivity in a 3.0-T scanner. However, when larger parts of the body such as the torso were imaged, this homogeneity could be reduced. To solve this problem, multi-channel excitation and RF shimming methods were proposed and investigated (17).

Although linear coils could solve the RF heating problem of implant leads in MRI, they caused twice as much average SAR as the quadrature coils. To reduce the SAR, computer simulations were performed. Instead of making a novel coil design, we calculated and optimized the field distributions of the coils, which provided us with 
important clues regarding the RF coil design. As can be seen from Fig. 6a-c, the electric field of an implantfriendly coil is actually a slightly distorted version of the field of a linear birdcage coil. This finding indicates that a slight modification of the geometry of a linear coil can significantly reduce the whole-volume average SAR without sacrificing homogeneity. An actual coil that has such field distribution characteristics needs to be built and further investigated.

The calculated field distributions can also be obtained using multi-channel transmit arrays. By controlling the currents in separate channels of a transmit array, the optimum field distributions can be approximated. As the number of the channels increases, the approximation will become more accurate. It is a new concept to use transmit arrays to reduce RF heating in implants. In the current study, we verified a similar concept using a linear birdcage coil, which can be directly applied to a twochannel transmit array system. However, the effectiveness of this multi-channel transmit array system remains to be investigated experimentally.

Knowing the location of the implant helps to reduce the average SAR. To locate the lead, the electric field near the implant can be set to zero, and low-SAR scans can be performed before the examination. The obtained information can be further used to calculate the optimum excitation currents of a transmit phased array.

In this work, the transmit field was optimized to reduce the RF heating of implants, and to obtain a uniform transmit sensitivity in the phantom. It should be noted that the transmit and receive problems are decoupled from each other. The receive chain can be optimized separately to achieve SNR enhancement, if necessary.

\section{CONCLUSIONS}

In this study, we showed that it is possible to modify the electric-field distribution of a RF coil to generate electric field-free zones in the body without significantly altering the transmit sensitivity. As supported by our experimental data, a linearly polarized birdcage coil can be safely used to scan patients with implants. To further alleviate the problems caused by the doubling of the whole-volume average SAR, implant-friendly EM field solutions with the desired transmit sensitivity and minimum SAR were simulated.

\section{APPENDIX}

Cylindrical mode solutions of Maxwell's equations (18) can be used to express the EM field of birdcage coils. The total electric field is the summation of the cylindrical modes that are shown below (14):

$$
\begin{gathered}
E_{z m n}=A_{m n} J_{m}\left(\beta_{\rho n} \rho\right) e^{j m \phi} e^{-j \beta_{z n} z} \\
E_{\phi m n}=\left[\frac{A_{m n}}{\rho \beta} J_{m}\left(\beta_{\rho n} \rho\right)+\frac{\omega \mu}{\beta} B_{m n} J_{m}^{\prime}\left(\beta_{\rho n} \rho\right)\right] e^{j m \phi} e^{-j \beta_{z n} z} \\
E_{\rho m n}=\left[\frac{B_{m n}}{\sigma^{\prime} \rho} J_{m}\left(\beta_{\rho n} \rho\right)-j A_{m n} J_{m}^{\prime}\left(\beta_{\rho n} \rho\right)\right] e^{j m \phi} e^{-j \beta_{z n} z}
\end{gathered}
$$

$$
\begin{aligned}
& H_{\rho m n}=\left[\frac{-m \beta^{2} A_{m n}}{\omega \mu \rho \beta_{\rho n}^{2}} J_{m}\left(\beta_{\rho n} \rho\right)-\frac{\beta_{z n} B_{m n}}{\beta_{\rho n}} J_{m}^{\prime}\left(\beta_{\rho n} \rho\right)\right] e^{j m \phi} e^{-j \beta_{z n} z} \\
& H_{\phi m n}=\left[\frac{-j m \beta_{z n} B_{m n}}{\rho \beta_{\rho n}^{2}} J_{m}\left(\beta_{\rho n} \rho\right)-\frac{\sigma^{\prime} A_{m n}}{\beta_{\rho n}} J_{m}^{\prime}\left(\beta_{\rho n} \rho\right)\right] e^{j m \phi} e^{-j \beta_{z n} z}
\end{aligned}
$$

where $A_{m n}$ and $B_{m n}$ are constants depending on the excitation, $J_{m}(x)$ is the Bessel function of order $m$ and $J_{m}{ }^{\prime}(x)$ denotes the derivative of the $m$ th order Bessel function. The $\boldsymbol{E}_{m n}$ matrix can be constructed for each mode as shown below:

The forward and reversed polarized magnetic field components for transmission are defined as:

$$
H_{\mathrm{f}}=\frac{\left(H_{\rho}-j H_{\phi}\right) e^{-j \phi}}{\sqrt{2}}, \quad H_{r}=\frac{\left(H_{\rho}+j H_{\phi}\right) e^{j \phi}}{\sqrt{2}}
$$

In the literature, $\mathrm{B} 1+$ is used to refer to the transmit sensitivity of a coil. B1+ is defined in the rotating frame of reference. In our work, we refer $H_{\mathrm{f}}$ as the transmit sensitivity. We solve our optimization problems by setting constraints on $H_{\mathrm{f}}$, which is defined in the phasor domain and proportional to $\mathrm{B} 1+$.

As a uniform transmit sensitivity is desired in a forward polarized birdcage coil, no " $\phi$ " and " $z$ " dependences are desired. Therefore, the field can be approximated by using the cylindrical expansion mode with $m=+1$ and $n=0$. Similarly, $m=-1$ and $n=0$ can be used to approximate a reverse polarized birdcage coil. The modal expressions can be further simplified by using the following Bessel function approximations:

if $\beta \rho<1$ then $J_{-1}(\beta \rho) \approx-\frac{\beta \rho}{2}, J_{1}(\beta \rho) \approx \frac{\beta \rho}{2}$ and $J_{0}(\beta \rho) \approx 1$

The approximate solutions for the forward polarized birdcage coil can be obtained as:

$$
\begin{aligned}
E_{z}=\frac{A \beta \rho}{2} e^{j \phi}, \quad E_{\phi}=\frac{\omega \mu B}{2 \beta} e^{j \phi}, \quad E_{\rho} & =\frac{-j B \omega \mu}{2 \beta} e^{j \phi}, \\
H_{f} & =-\frac{A \beta}{\omega \mu_{0}}, \quad H_{r}=0
\end{aligned}
$$

Note that the $\beta \rho<1$ condition implies that the radius of the object is much smaller than the wavelength. As can be seen in the expression of $H_{\mathrm{f}}$, the transmit sensitivity is independent from the position of the coil. The constant $B$ does not have any effects on the forward polarized magnetic field and only contributes to the electric field. Therefore, for the most efficient excitation (birdcage quadrature excitation), $B$ should be equal to zero to minimize SAR, which results in the simplified expression of the following form:

$$
E_{\phi}=E_{\rho}=0, \quad E_{z}=\frac{-H_{f} \omega \mu_{0} \rho}{2} e^{j \phi}, \quad H_{f}=-\frac{A \beta}{\omega \mu_{0}}, \quad H_{r}=0
$$


With the same approximations, field expressions of a reverse polarized birdcage coil can be expressed as:

$$
\begin{aligned}
& E_{\phi}=E_{\rho}=0, \quad E_{z}=\frac{H_{f} \omega \mu_{0} \rho}{2} e^{-j \phi}, \quad H_{\mathrm{f}}=, \\
& H_{r}=-\frac{A \beta}{\omega \mu_{0}}
\end{aligned}
$$

By including the expressions of the forward and reverse polarized fields, one can express the approximate field components of a linear coil as:

$$
\begin{array}{r}
E_{\phi}=E_{\rho}=0, \quad E_{z}=-H_{f} j \omega \mu_{0} \rho \sin \phi, \quad H_{\mathrm{f}}=-\frac{A \beta}{\omega \mu_{0}}, \\
H_{r}=-\frac{A \beta}{\omega \mu_{0}}
\end{array}
$$

\section{REFERENCES}

1. Baker KB, Tkach JA, Nyenhuis JA, Phillips M, Shellock FG, Gonzalez-Martinez J, Rezai AR. Evaluation of specific absorption rate as a dosimeter of MRI-related implant heating. J Magn Reson Imaging 2004;20:315-320.

2. Sommer T, Vahlhaus C, Lauck G, von Smekal A, Reinke M, Hofer U, Block W, Traber F, Schneider C, Gieseke J, Jung W, Schild H. MR imaging and cardiac pacemakers: in-vitro evaluation and in-vivo studies in 51 patients at 0.5 T. Radiology 2000;215:869-879.

3. Yeung CJ, Atalar E. A Green's function approach to local rf heating in interventional MRI. Med Phys 2001;28:826-832.

4. Yeung CJ, Susil RC, Atalar E. RF safety of wires in interventional MRI: using a safety index. Magn Reson Med 2002;47:187-193.

5. Nordbeck P, Weiss I, Ehses P, Ritter O, Warmuth M, Fidler F, Herold V, Jakob PM, Ladd ME, Quick HH, Bauer WR. Measuring RF-induced currents inside implants: impact of device configuration on MRI safety of cardiac pacemaker leads. Magn Reson Med 2009;61: $570-578$.

6. Ferhanoglu O, Eryaman Y, Atalar E. MRI Compatible pacemaker leads. Proc Intl Soc Mag Reson Med 2005;13:963.

7. Ladd ME, Quick HH. Reduction of resonant RF heating in intravascular catheters using coaxial chokes. Magn Reson Med 2000;43: 615-619.

8. Gray RW, Bibens WT, Shellock FG. Simple design changes to wires to substantially reduce MRI-induced heating at $1.5 \mathrm{~T}$ : implications for implanted leads. Magn Reson Imaging 2005;23:887-891.

9. Nordbeck P, Fidler F, Weiss I, Warmuth M, Friedrich MT, Ehses P, Geistert W, Ritter O, Jakob PM, Ladd ME, Quick HH, Bauer WR. Spatial distribution of RF-induced E-fields and implant heating in MRI. Magn Reson Med 2008;60:312-319.

10. Yeung CJ, Susil RC, Atalar E. RF heating due to conductive wires during MRI depends on the phase distribution of the transmit field. Magn Reson Med 2002;48:1096-1098.

11. Jin J, Chen J. On the SAR and field inhomogeneity of birdcage coils loaded with the human head. Magn Reson Med 1997;38:953-963.

12. Glover GH, Hayes CE, Pelc NJ, Edelstein WA, Mueller OM, Hart HR, Hardy CJ, O’Donnell M, Barber WD. Comparison of linear and circular polarization for magnetic resonance imaging. J Mag Res 1985;64: 255-270.

13. Lattanzi R, Sodickson DK, Grant AK, Zhu Y. Electrodynamic constraints on homogeneity and radiofrequency power deposition in multiple coil excitations. Magn Reson Med 2009;61:315-334.

14. Celik H, Eryaman Y, Altintas A, Abdel-Hafez IA, Atalar E. Evaluation of internal MRI coils using ultimate intrinsic SNR. Magn Reson Med 2004;52:640-649.

15. Ocali O, Atalar E. Ultimate intrinsic signal-to-noise ratio in MRI. Magn Reson Med 1998;39:462-473.

16. Akin B, Eryaman Y, Atalar E. A method for phantom conductivity and permittivity measurements. ESMRMB;2009.

17. Vernickel P, Roschmann P, Findeklee C, Ludeke KM, Leussler C, Overweg J, Katscher U, Grasslin I, Schunemann K. Eight-channel transmit/receive body MRI coil at 3T. Magn Reson Med 2007;58: 381-389.

18. Foo TK, Hayes CE, Kang YW. An analytical model for the design of RF resonators for MR body imaging. Magn Reson Med 1991;21:165-177. 